\title{
Defect-induced phase transition in the asymmetric simple exclu- sion process
}

\author{
Johannes Schmidt ${ }^{1}$, Vladislav Popkov ${ }^{1,2}$ and Andreas Schadschneider ${ }^{1}$ \\ 1 Institut für Theoretische Physik, Universität zu Köln, 50937 Köln, Germany \\ 2 CSDC Università di Firenze, via G.Sansone 1, 50019 Sesto Fiorentino, Italy
}

PACS 02.50.Ey - Stochastic processes

PACS 05.70.Fh - Phase transitions: general studies

PACS 05.60.-k - Transport processes

\begin{abstract}
We reconsider the long-standing question of the critical defect hopping rate $r_{c}$ in the one-dimensional totally asymmetric exclusion process (TASEP) with a slow bond (defect). For $r<r_{c}$ a phase separated state is observed due to queuing at the defect site whereas for $r \geq r_{c}$ the defect site has only local effects on the stationary state of the homogeneous system. Mean-field theory predicts $r_{c}=1$ (when hopping rates outside the defect bond are equal to 1 ) but numerical investigations seem to indicate $r_{c} \approx 0.80(2)$. Here we improve the numerics to show that $r_{c}>0.99$ and give strong evidence that indeed $r_{c}=1$ as predicted by mean-field theory, and anticipated by recent theoretical findings.
\end{abstract}

Introduction. - Despite much progress in recent years, our understanding of nonequilibrium stationary ' states is far from complete. This especially concerns the effects of disorder and defects in driven diffusive systems. ' Although it is well established that in driven systems already a localized defect can have a global influence on the behavior, many open questions remain. Since e.g. no analogue of the Harris criterion [1] is known for nonequilibrium situations no general statements on the influence of weak disorder on the critical behavior can be made [2].

Surprisingly even for the simplest model of driven diffusion, the totally asymmetric exclusion process (TASEP), the precise influence of a single defect has not been fully clarified for a long time. It is well-known since the seminal work of Janowsky and Lebowitz [3, 4] that such a defect has not just a local effect, but changes the nature of the stationary state dramatically (for reviews, see e.g. [5, 6]). What is not clear up to now is whether a finite critical strength of the defect is required to create global effects. Mean-field theory predicts a global influence already for arbitrarily small defect strengths whereas the most accurate numerical investigations up to date [7] show strong indications that a finite defect strength is required.

Recently the problem has been newly addressed in the mathematical literature, in a series of works 8 11. In [8], based on analytical arguments from series expansions and results for related systems (e.g. directed polymers) it was argued that an arbitrarily small defect in a TASEP with open boundaries will have global effects, e.g. on the current and on the density profile. In 9, the authors claim to have proved rigorously, that the steady current in TASEP with a slow bond is always affected for any nonzero defect strength.

In view of these new findings, the numerical studies predicting finite critical blockage strength, appear even more paradoxical, with the $r_{c}=1$ problem finally settled. It remains to understand if the effects of the slow bond are so weak that they cannot be observed in numerics, which would make the beautiful theoretical result a pure theoretical construction without any practical content.

It is the purpose of this letter to show that this is not the case, and to provide detailed results from highly accurate Monte Carlo simulations which strongly support the theory. Moreover, we also resolve the apparent numerical paradox, revisiting previous numerical studies and pointing out exactly where the error of the previous numerical studies was.

Model. - The totally asymmetric simple exclusion process (TASEP) is a paradigmatic model of nonequilibrium physics (for reviews, see e.g. [12 18]) describes interacting (biased) random walks on a discrete lattice of $N$ sites, where an exclusion rule forbids occupation of a site by more than one particle. A particle at site $k$ moves to 


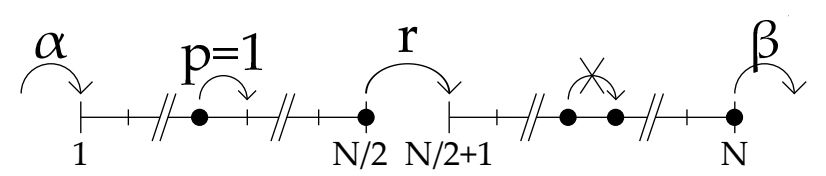

Fig. 1: Open TASEP with a slow bond $(r<1)$ in the middle.

site $k+1$ at rate $p$ if site $k+1$ is not occupied by another particle. The boundary sites $k=1$ and $k=N$ are coupled to particle reservoirs. If site 1 is empty, a particle is inserted at rate $\alpha$. A particle on site $N$ is removed from the system at rate $\beta$. Sites are updated using randomsequential dynamics. Throughout the paper, we will set $p=1$.

Here we consider a system of two TASEPs of length $N / 2$ coupled by a slow bond between sites $N / 2$ and $N / 2+1$ with reduced hopping rate $r \leq p$ (Fig. (1). This is equivalent to a TASEP of $N$ sites with a defect site in the middle and defect strength $p-r$.

For periodic boundary conditions this problem has been analyzed by Janowsky and Lebowitz [3, 4]. Below a critical rate $r_{c}$ they found a phase separation into high and low density regions due to queuing at the defect site. The two phases are separated by a shock (domain wall). The phase separation is also reflected in the current-density relation (fundamental diagram) which shows a densityindependent current at intermediate densities due to the current-limiting effect of the slow bond. A mean-field theory that neglects correlations at the defect site predicts that $r_{c}=1$ [3], i.e. an arbitrarily small defect leads to a phase separated stationary state. This is supported by series expansions obtained from exact results for small systems [4. Exact results have been obtained for the case of sublattice-parallel update with deterministic bulk hopping by Bethe Ansatz [19] and matrix-product Ansatz [20]. Also in this case $r_{c}=1$.

For open boundary conditions, a mean-field treatment of the TASEP with a defect in the middle of the system yields $r_{c}=1$ [21. Later Ha et al. [7] studied the problem numerically (with rates $p=1, \alpha=\beta=1 / 2$ ). They suggested that $r_{c}=0.80(2)$, see next section for details.

Due to its relevance e.g. for intracellular transport, recently the open TASEP with several defects has attracted some attention, see e.g. [22 33].

Simulations. - In order to analyze the system with a defect we perform Monte Carlo (MC) simulations for an open TASEP on a chain of large size $(N \leq 200.000)$, to minimize finite-size effects as much as possible. Both bulk hopping rates and boundary hopping rates are chosen equal to 1 . This corresponds to a point in the maximal current phase which is characterized by a spatially homogeneous steady state with bulk density of particles $\rho_{\text {bulk }}=1 / 2$ and the current $j(\infty)=\rho_{\text {bulk }}\left(1-\rho_{\text {bulk }}\right)=1 / 4$ in the limit of an infinite system size [13].

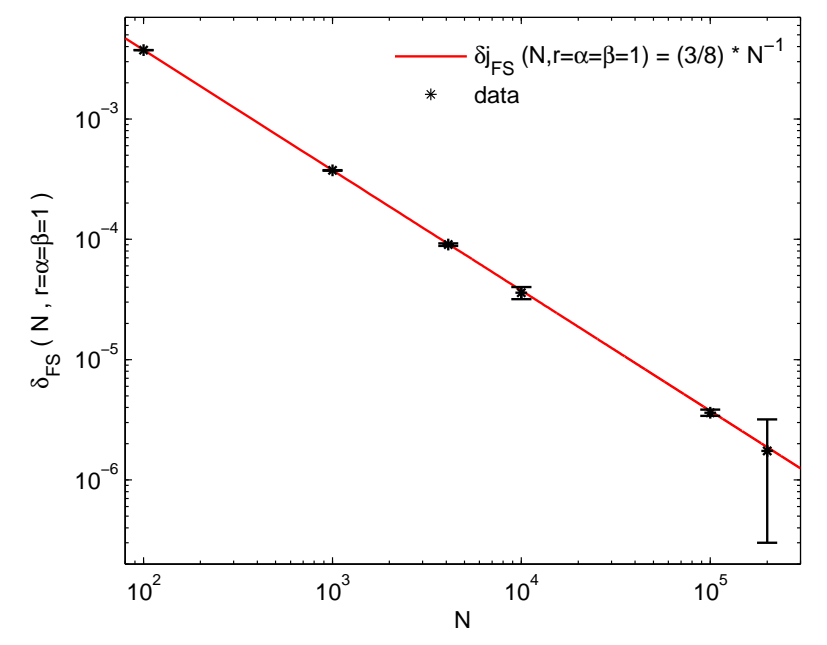

Fig. 2: Finite-size corrections of the steady current for a homogeneous model without slow bond. Error bars show the $99 \%$ confidence bound, the red line marks the exact leading finite-size correction in $1 / N$.

Initially $N / 2$ particles are distributed randomly within a homogeneous chain without a defect. Then, the relaxation of the system is performed for $100 N^{2}$ single Monte Carlo updates 34, according to the dynamical rules for $\alpha=\beta=$ 1.

After the initial relaxation, the weak bond is introduced in the middle of the system, meaning that a particle hop from site $k=N / 2$ to $N / 2+1$ occurs with reduced rate $r<1$, see Fig. 1. Then the system is relaxed further for $100 N^{2}$ single Monte Carlo updates and the average current is recorded. It can be written as

$$
j_{\mathrm{FS}}(r, N, \alpha, \beta)=j(r, \infty)+\delta_{\mathrm{FS}}(r, N, \alpha, \beta)
$$

where $\delta_{\mathrm{FS}}$ are finite-size corrections. The relaxation to steady state is controlled by a comparison of the finitesize corrections of the current measured numerically with the theoretically-predicted value, see Fig. 2

We aim at determining a lower bound for the critical $r_{c}$ at which the phase separation starts. It is well-known 35. that the leading finite-size corrections to the current of the homogeneous TASEP for $\alpha=\beta=1$ are positive,

$$
\begin{aligned}
j_{\mathrm{FS}}(r=1, N, \alpha=1, \beta=1) & =j(r=1, \infty)+\frac{3}{8 N}+O\left(N^{2}\right) \\
& >j(r=1, \infty) .
\end{aligned}
$$

Therefore, if for some defect hopping rate $r_{0}$ the steady current within the error bars is smaller than its limiting value, $j\left(r_{0}, N\right)<1 / 4$, this would definitely mean that $r_{c}>r_{0}$. The lower bound $r^{*}$ for $r_{c}$ is then calculated as the point where $j\left(r^{*}, N\right)=1 / 4$, accounting also for error bars, see Fig. 3 Note that our reasoning does not involve any a priori assumptions except from the positiveness of finite-size corrections to the current. In this way, we obtain 


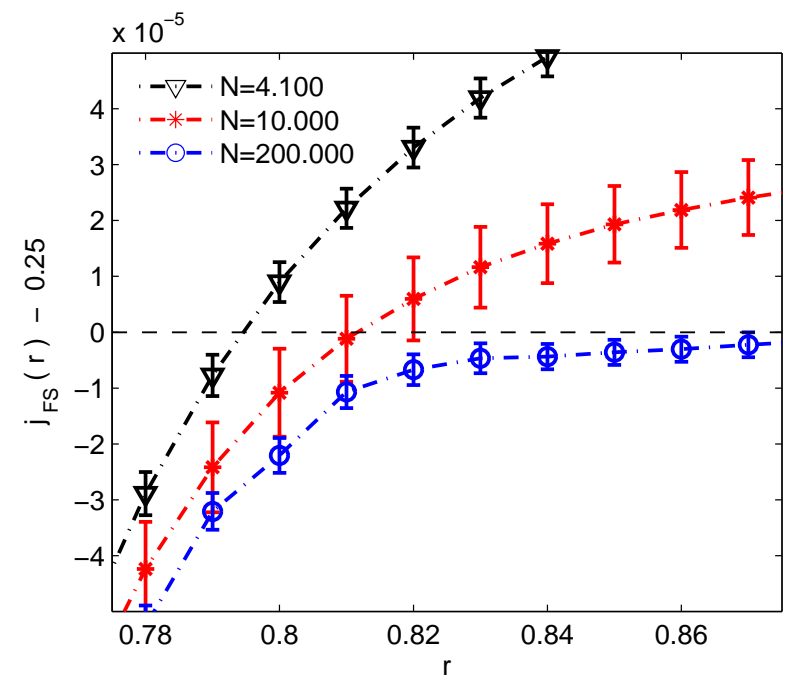

Fig. 3: Finite-size current $j_{\mathrm{FS}}$ (see eq. (1)) versus defect rate $r$ for $\alpha=\beta=1$. With $j(r) \leq j_{F S}(r)$ the plot shows $r_{c}>0.86$ which is significantly larger than $r_{c}=$ 0.80 (2) suggested in 7. Currents are averaged over all sites with $7.5 \cdot 10^{7}-5 \cdot 10^{8}$ histories. Error bars show the $99 \%$ confidence interval.

a lower bound for $r_{c}$ which depends on the system size. For larger system size, finite-size corrections are smaller and better estimates for the lower bound can be made, see Fig. 3. For system size $N=2 \cdot 10^{5}$ we obtain the following lower bound estimate,

$$
r_{c}>0.86 .
$$

It is difficult to improve the lower bound (3) by a further increase of the system size $N$, because much larger system sizes are not numerically accessible. However, already the lower bound value 0.86 definitely contradicts the result of Ha et al. 7], where $r_{c}=0.80(2)$ was found. Their estimate was based on a different argument. In order to understand the reason for the contradiction, we repeated Monte Carlo simulations with the same parameters as in [7, and in particular using the much smaller system size of $N=4100$.

The key quantity analyzed by Ha et al., is defined as

$$
\Delta_{b} \propto\left|\rho_{\text {bulk,segment }}-\frac{1}{2}\right|
$$

or

$$
\Delta_{b}=2 \sqrt{j(r=1, N)-j(r, N)} .
$$

It is assumed to obey the scaling form

$$
\Delta_{b} \sim\left(r_{c}-r\right)^{-\beta}
$$

near the phase boundary. Then, the best straight line fit on the double logarithmic plot of $\Delta_{b}$ versus $r_{c}-r$, has lead to the conclusion $r_{c}=0.80(2)$. We repeated

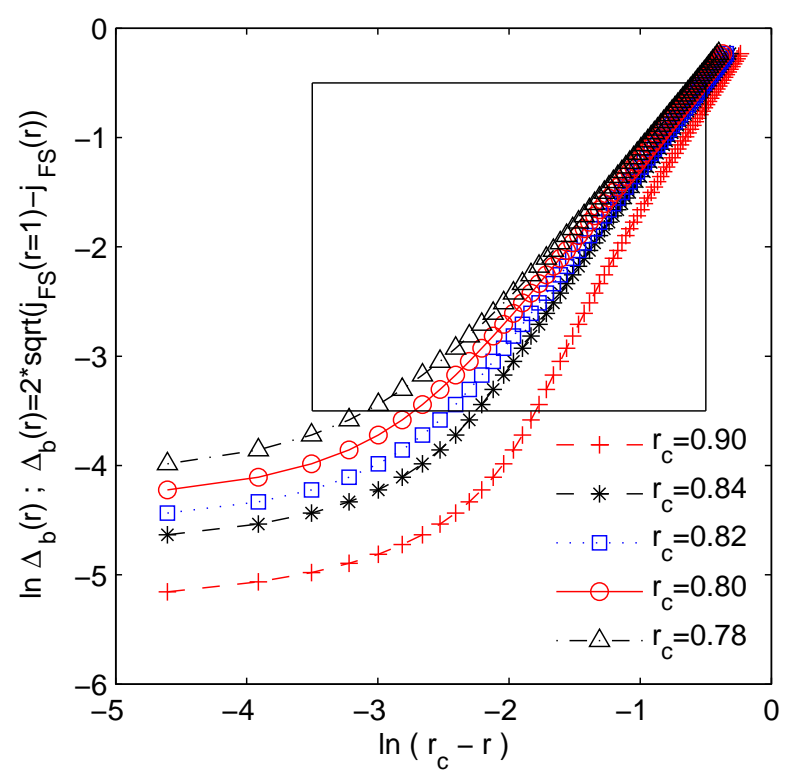

Fig. 4: Double logarithmic plot of $\Delta_{b}$ versus $\left(r_{c}-r\right)$. Parameters are $N=4100, \alpha=\beta=0.5$. The box indicates the regime plotted in Fig. 4a of [7. Averaging over all sites of the system and over $3 \cdot 10^{8}$ histories is performed. Statistical errors are smaller than the symbol size.

the relevant Monte Carlo simulations for the parameters chosen in [7. Fig. 4 shows the double logarithmic plot of $\Delta_{b}$ versus $r_{c}-r$. The square window corresponds to the area shown in the original paper, see Fig. 4a of [7]. We can see that what might look as a straight line inside the window, certainly fails to straighten outside the window. Thus the conclusion of [7] of a phase transition at $r_{c}=$ $0.80(2)$ is not justified.

It is instructive at this point to stress the importance of a choice of an adequate random generator to perform the Monte Carlo update. This choice is crucial for producing high quality Monte Carlo data 36. In Fig. 5 , Monte Carlo data for the current, produced by different random number generators, are compared and show systematic differences. For our simulations throughout this paper we are using Mersenne-Twister-generator which is known for producing high quality pseudo-random numbers. In Fig. 5 it is seen that using the most common Park and Miller new minimal standard linear congruential generator 37 leads to a systematic overestimation of the current, and might consequently lead to wrong conclusions in the subtle TASEP blockage problem.

We also note, that the point $\alpha=\beta=1 / 2$ as chosen for studying the blockage problem in [7 lies exactly at the phase boundary between the maximal current, low density and high density phases 1218 . This may lead to further complications and an additional reduction of the steady current due to fluctuations. We stress that for our study we choose $\alpha=\beta=1$, i.e. a point well inside the 


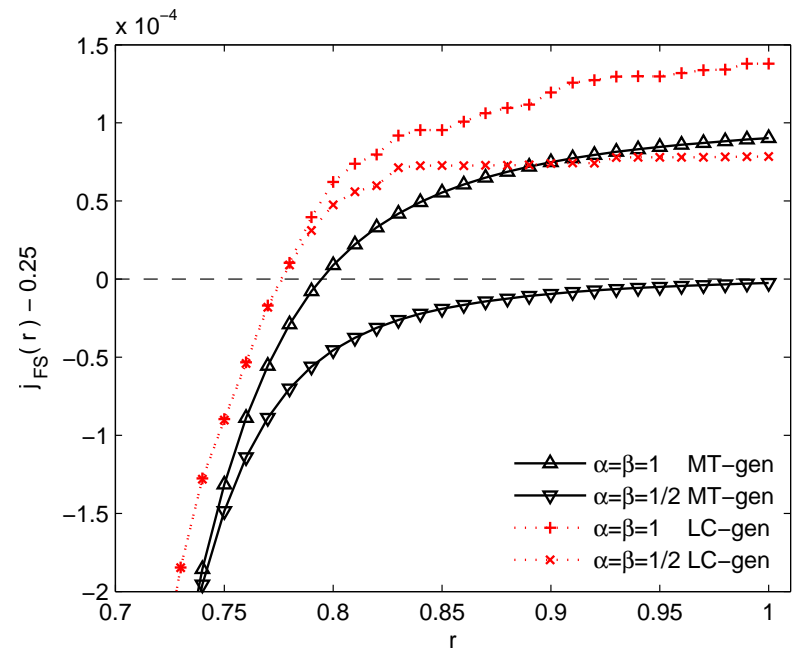

Fig. 5: Finite-size corrections of the current eq. (10), for a different choices of a random number generators. Parameters: $N=4100$. Triangles correspond to Mersenne Twister random number generator and crosses correspond to a standard Park and Miller new minimal standard (LC-gen, linear congruential) random number generator 37. Averaging of the current over all bonds and over $3 \cdot 10^{8}-5 \cdot 10^{8}$ histories is performed. Statistical errors are smaller than the symbol size.

maximal current phase far from the boundaries with the low density TASEP phase $(\alpha=1 / 2)$ and the high density TASEP phase $(\beta=1 / 2)$.

Effects of a defect in finite systems: parallel evolution. - As is already mentioned, both mean-field theory and series expansions arguments hint at $r_{c}=1$. Assuming the existence of an essential singularity at $r_{c}=1$, $j(1)-j(r) \sim \exp (-a /(1-r))$ [8], further improvement of the lower bound for the weak bond problem by an increase of the system size is a hopeless enterprise: e.g. a numerical proof $\left.r_{c}>0.9, r_{c}>0.95, r_{c}>0.99\right)$ with the direct method (see Fig. 3) would require $N>10^{10}, N>10^{22}$, $N>10^{147}$ respectively.

Instead of increasing the system size, we address the problem of a critical blockage strength in different way by measuring how a TASEP responds to a slow bond, as discussed below.

After the pre-relaxation performed on the homogeneous system as described above, we make two copies of the system configuration. Then a slow bond is introduced in one copy whereas the other remains homogeneous. Both copies evolve in time according to the same protocol, i.e. using the same set of random numbers for both systems. The averaged density profiles of both copies are compared after sufficiently large relaxation time. Due to the defect site, one expects a density gradient forming locally in the vicinity of the blockage for any $r<1$. If the disturbance remains local, the state of the system far from the blockage will not change, with respect to a homogeneous system. In contrast, a non-local disturbance spreading to the whole system would lead to a reduction of the global current and to phase separation. Thus the current and the density profile are sensitive probes for the effects of the slow bond and for the possible occurrence of a phase separated state.

Performing extensive MC simulations we are able to see a non-local effect of the blockage up to $r=0.99$, both in steady current and in local particle density far away from the blockage, see Fig. 6. Consequently, a presence of the weak bond has a small but systematic effect on both the current and the local density $n_{k}$ far away from the blockage site. This shows that the blockage produces perturbations which do not remain local, but spread over the bulk.

Conclusion. - We have revisited a long-standing problem of a TASEP with a weak bond. Since the effects are small for small defect strength, this is a subtle problem that requires high numerical accuracy in simulations. We have shown that here even the choice of the random number generator is crucial. By Monte Carlo simulations performed on large systems (up to 200.000 sites), we have established a new lower bound for the critical defect strength leading to phase separation. For the current, we could clearly show a reduction compared to the value $j(r=1)=1 / 4$ of the homogeneous system for defect hopping rates up to $r=0.86$. Therefore we conclude that $r_{c}>0.86$. Studying a parallel evolution of two initially identical systems, with and without a weak bond, we find systematic global effects induced by the weak bond for defect hopping rate up to $r \leq 0.99$. Our study supports the hypothesis that the critical blockage hopping rate is $r_{c}=1$, and definitely rules out a previously obtained critical value $r_{c}=0.80(2)$. This indicates that the meanfield theory prediction is indeed correct which is important since it is used quite frequently also in more complex situations, like flows on networks [38,42] or as effective models for highway traffic near ramps 43 .

$$
* * *
$$

We thank Meeson Ha for providing background information on their work 7 and Gunter M. Schütz for discussions. Financial support by the DFG under grant SCHA $636 / 8-1$ is gratefully acknowdledged.

\section{REFERENCES}

[1] A.B. Harris: J. Phys. C 7, 1671 (1974)

[2] R. Stinchcombe: J. Phys.: Condens. Matter 14, 1473 (2002)

[3] S.A. Janowsky, J.L. Lebowitz: Phys. Rev. A 45, 618 (1992)

[4] S.A. Janowsky, J.L. Lebowitz: J. Stat. Phys. 77, 35 (1994)

[5] J. Krug: Braz. J. Phys. 30, 97 (2000)

[6] M. Barma: Physica A 372, 22 (2006) 

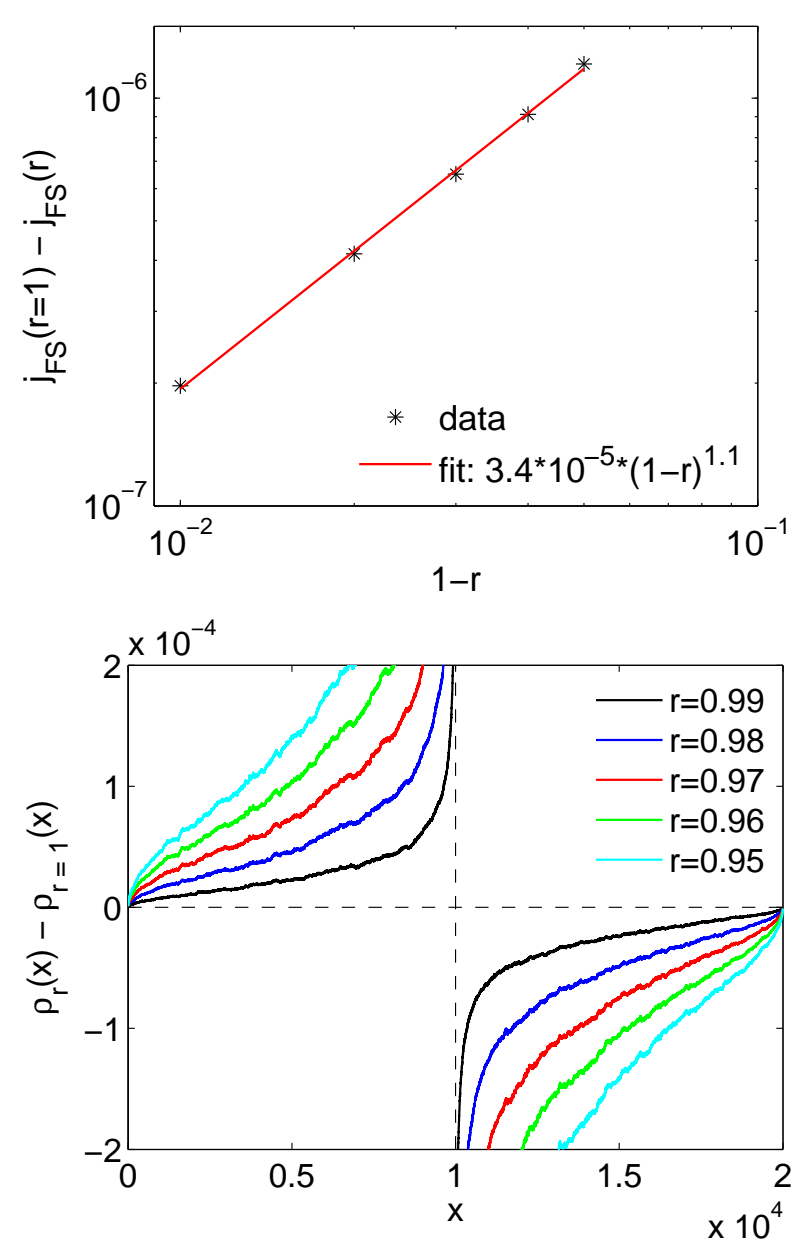

Fig. 6: Steady current differences (top) and density differences (bottom) between the parallel evolving copies of the same system (with and without defect). Averaging over $10^{9}$ histories is performed for systems with $N=2 \cdot 10^{4}$ sites. For the currents (top) error bars (not shown) are of the order of the symbol size. For the density differences (bottom), statistical errors are in the range from $10^{-8}$ close to the reservoirs up to $4.6 \cdot 10^{-6}$ in the vicinity of the defect.

[7] M. Ha, J. Timonen, M. den Nijs: Phys. Rev. E 58, 056122 (2003)

[8] O. Costin, J. L. Lebowitz, E. R. Speer, A. Troiani: Bulletin of the Institute of Mathematics Academia Sinica 8, 49 (2013)

[9] R. Basu, V. Sidoravicius and A. Sly, arXiv:1408.3464

[10] J.Calder, J. Stat. Phys. 158, 903 (2015)

[11] B. Scoppola, C. Lancia e R. Mariani, arXiv:1409.0268

[12] T.M. Liggett: Stochastic Interacting Systems: Contact, Voter and Exclusion Processes, Springer, New York (1999)

[13] G.M. Schütz, in Phase Transitions and Critical Phenomena vol 19., C. Domb and J. L. Lebowitz Ed., Academic Press, San Diego (2001)

[14] R. K. P. Zia and B. Schmittmann, J. Stat. Mech. (2007)
P07012

[15] A. Schadschneider, D. Chowdhury, K. Nishinari: Stochastic Transport in Complex Systems: From Molecules to Vehicles, Elsevier Science, Amsterdam (2010)

[16] P.L. Krapivsky, S. Redner, E. Ben-Naim: A Kinetic View of Statistical Physics, Cambridge University Press, Cambridge (2010)

[17] R.A. Blythe, M.R. Evans: J. Phys. A: Math. Gen. 40, R333 (2007)

[18] B. Derrida: J. Stat. Mech. (2007) P07023

[19] G. Schütz: J. Stat. Phys. 71, 471 (1993)

[20] H. Hinrichsen, S. Sandow: J. Phys. A 30, 2745 (1997)

[21] A.B. Kolomeisky: J. Phys. A 31, 1153 (1998)

[22] G. Tripathy, M. Barma: Phys. Rev. E 58, 1911 (1997)

[23] T. Chou, G.W. Lakatos: Phys. Rev. Lett. 93, 198101 (2004)

[24] G.W. Lakatos, J. O'Brien, T. Chou: J. Phys. A 39, 2253 (2006)

[25] R.J. Harris, R.B. Stinchcombe: Phys. Rev. E 70, 016108 (2004)

[26] C. Enaud, B. Derrida: Europhys. Lett. 66, 83 (2004)

[27] R. Juhasz, L. Santen, F. Igloi: Phys. Rev. E 74, 061101 (2006)

[28] P. Pierobon, M. Mobilia, R. Kouyos, E. Frey: Phys. Rev. E 74, 031906 (2006)

[29] J.J. Dong, B. Schmittmann, R.K.P. Zia: J. Stat. Phys. 128, 21 (2007)

[30] M.E. Foulaadvand, S. Chaaboki, M. Saalehi: Phys. Rev. E 75, 011127 (2007)

[31] P. Greulich, A. Schadschneider: Physica A387, 1972 (2008)

[32] P. Greulich, A. Schadschneider: J. Stat. Mech. (2008) P04009

[33] P. Greulich, A. Schadschneider: Phys. Rev. E 79, 031107 (2009)

[34] A single Monte Carlo update consists in choosing a bond at random, and updating its configuration according to dynamical rules.

[35] B. Derrida, M. Evans: J. Physique I 3, 311 (1993)

[36] P. L'Ecuyer, R. Simard: ACM Trans. Math. Softw. 33, 22 (2007)

[37] S. K. Park, K. W. Miller: Commun. ACM 31, 1192 (1988)

[38] B. Embley, A. Parmeggiani, N. Kern: Phys. Rev. E 80, 041128 (2009)

[39] T. Ezaki, K. Nishinari: J. Stat. Mech. (2012) P11002

[40] A. Raguin, A. Parmeggiani, N. Kern: Phys. Rev. E 88, 042104 (2013)

[41] I. Neri, N. Kern, A. Parmeggiani: New J. Phys. 15, 085005 (2013)

[42] Y. Baek, M. Ha, J. Jeong: Phys. Rev. E 90, 062111 (2014)

[43] G. Diedrich, L. Santen, A. Schadschneider, J. Zittartz: Int. J. Mod. Phys. C 11, 335 (2000) 